\title{
INFLORESCENCE SCENTS OF CALENDULA MARITIMA, CALENDULA SUFFRUTICOSA SUBSP. FULGIDA, AND THEIR HYBRID
}

\author{
Pietro Zito, ${ }^{1, *}$ Francesca Tavella, ${ }^{*}$ Maurizio Sajeva, ${ }^{*}$ Francesco Carimi, + and Stefan Dötterlł \\ *Department of Biological, Chemical, and Pharmaceutical Sciences and Technologies, University of Palermo, Via Archirafi, \\ 18, 90123 Palermo, Italy; Institute of Biosciences and Bioresources, Division of Palermo, National Research \\ Council of Italy-Corso Calatafimi, 414, 90129 Palermo, Italy; and ¥Department of Biosciences, \\ Plant Ecology, University of Salzburg, Hellbrunner Straße 34, 5020 Salzburg, Austria
}

Editor: Félix Forest

\begin{abstract}
Premise of research. Hybridization is an important driver of plant evolutionary processes. By attracting the same pollinators to different species, floral scents may be involved in the formation of hybrids and breakdown of species boundaries. In contrast, by attracting a different suite of pollinators to hybrids and their parents, floral scents are believed to contribute to speciation processes initiated by hybridization events. Scents may or may not differ between the hybrids and their parents, but little is known about the scent chemistry of parental species and their hybrids.
\end{abstract}

Methodology. We studied the inflorescence scents of parental Calendula maritima and C. suffruticosa subsp. fulgida (henceforth, C. fulgida) and a morphologically intermediate hybrid. Scents were collected by dynamic headspace and analyzed by thermal desorption-gas chromatography/mass spectrometry. Insects visiting inflorescences of the three taxa were also captured.

Pivotal results. Calendula maritima and C. fulgida emitted different absolute amounts of scent; the hybrid released intermediate amounts of scent. The scents of the parental and hybrid taxa were all dominated by monoterpenes, with several compounds in common among the taxa. Nevertheless, the three taxa showed differences in qualitative and semiquantitative scent patterns. Calendula maritima emitted more and a higher amount of sesquiterpenes than the other taxa. The hybrid was overall more similar in scent properties to C. fulgida than it was to C. maritima.

Conclusions. The overlap in scent compounds among the taxa may be responsible for attraction of the same insect pollinators, resulting in interspecific pollen transfer between the parents and the formation of hybrids. Indeed, preliminary observations revealed that all three taxa are visited by Panurgus siculus bees (Andrenidae).

Keywords: Asteraceae, hybridization, marigolds, Panurgus, pollination, volatile organic compounds.

\section{Introduction}

Plants pollinated by animals have various reproductive isolation mechanisms acting before and/or after pollination that limit hybridization (Schatz et al. 2010). However, hybridization is an important driver of plant evolution (Waser 2001; Hegarty and Hiscock 2005; Schatz et al. 2010) and is especially common in cases where closely related species are syntopic (Bischoff et al. 2014). If such species overlap in their bloom periods and share the same pollinator species, and if specific pollinator individuals move among flowers of such species, hybridization can occur (Schatz et al. 2010; Bischoff et al. 2014). Floral scents have important roles in guiding pollinators to their host flowers and thus may be involved in the formation of hybrids (Cortis et al. 2009).

\footnotetext{
1 Author for correspondence; email: zitopietro@gmail.com.
}

Manuscript received September 2017; revised manuscript received December 2017; electronically published May 10, 2018.
Natural hybrids may or may not differ in scent properties from their parents, resulting in different reproductive scenarios (Bischoff et al. 2014). If the hybrids' scent is intermediate compared with their parents, it can promote backcrossing with both parental species and gene introgression (Salzmann and Schiestl 2007; Cortis et al.2009). In cases where the hybrids have a scent that differs from the scent of their parental species, scents may be involved in speciation processes (Vereecken et al. 2010). Finally, as inferred by Bischoff et al. (2014), if the hybrids are more similar to one parent, unidirectional backcrossing may occur.

This study focuses on a hybrid and its two parental taxa in Mediterranean Calendula, a genus being at least partially selfincompatible and depending on insect pollen vectors for outcrossing (Plume et al. 2013). In particular, our study plants were the yellow-flowered C. maritima Guss, the orange-flowered C. suffruticosa Vahl. subsp. fulgida (Raf.) Guadagno (henceforth, C. fulgida), and an intermediate form (hybrid) that has leaf properties in common with C. maritima (leaves entire and fleshy) and inflorescence properties in common with C. fulgida 
(inflorescences orange or pale orange; Plume et al. 2013). Early in 1919, Lanza reported that hybrids between C. maritima and C. fulgida were rare. Nowadays, the hybrid is widespread, probably because of human activities (see also Kölreuter 1761), which brought soil with seeds of C. fulgida to areas where C. maritima grows. This enabled the two parental species to occupy the same habitat and made frequent hybridization events possible.

The aim of this study is to gather data on inflorescence volatiles produced by the parental taxa (C. maritima and C. fulgida) and their interspecific hybrid in a zone where the three taxa cooccur. We also discussed preliminary data on insects visiting the inflorescences. Based on the findings of floral morphology, floral color, and frequent hybridization events, we expect that inflorescence scents do not differ between parents, nor between the parents and their hybrid, and that the taxa share inflorescence visitors/pollinators.

\section{Material and Methods}

\section{Plant Taxa}

Calendula maritima Guss. is an annual or short-lived perennial herb that is $20-40 \mathrm{~cm}$ tall. From mainly May to June it produces yellow inflorescences, though inflorescences can be found throughout the year (De Montmollin and Strahm 2005). Calendula maritima is an endemic species that grows in Sicily (near Trapani) and in some surrounding islets (Isola Grande dello Stagnone, La Formica, Maraone, and Colombaia; Plume et al. 2013). It is found on sand and rock in open areas near the sea and in Posidonia oceanica (L.) Delile seagrass litter, called "banquettes" (Plume et al. 2013). Its natural habitat is under threat from urban development as well as by competition with invasive species (e.g., Carpobrotus edulis (L.) N.E. Br.). To date, it is among "the top 50 Mediterranean island plants" (De Montmollin and Strahm 2005), and it is categorized as critically endangered according to the International Union for Conservation of Nature Red List (Troìa and Pasta 2006).

Calendula fulgida Raf. is a perennial erect herb ranging from 20 to $40 \mathrm{~cm}$ tall. It produces solitary orange inflorescences in the same period of the year as C. maritima (Plume et al. 2013). Its distribution is wider than in C. maritima and includes, besides Sicily, southern mainland Italy (Calabria), Tuscany (Rizzotto 2003), Malta, and Morocco (Fennane and Tattou 1998). It grows in disturbed habitats (e.g., fallow fields and roadcuts) and is not endangered.

Pollen transfer between C. maritima and C. fulgida occurs, resulting in the formation of hybrids (Plume et al. 2013). These hybrids have two intermediate morphologies. In one intermediate form, used for the present work, the leaves are entire and fleshy, as in C. maritima, while the inflorescences are orange or pale orange, as in C. fulgida; in a second form, the vegetative features are intermediate between the two parents but closer to C. fulgida, whereas the inflorescences are yellow, as in C. maritima. The form used for the present work is more common than the other form and is the only one that co-occurs with C. maritima. The flowering periods of the studied hybrid and its parents overlap, mainly from May to June, and intermediate forms frequently occur in natural habitats (Plume et al.
2013). Vouchers were deposited at the herbarium of the Department of Biological, Chemical, and Pharmaceutical Sciences and Technologies of the University of Palermo.

\section{Collection of Scents}

Inflorescence scents of C. maritima, C. fulgida, and their hybrid were collected in May 2015 from plants growing in their natural habitat, at a site known as Stele di Anchise (lat. $38^{\circ}$ $03^{\prime} 21.3^{\prime} \mathrm{N}$, long. $12^{\circ} 33^{\prime} 17.9^{\prime} \mathrm{E}$, district of Trapani, Sicily). For each taxon, the scent of eight different plants in full bloom was sampled by dynamic headspace. To sample the volatile organic compounds (VOCs) each inflorescence was enclosed in an oven bag $(20 \mathrm{~cm} \times 13 \mathrm{~cm}$, Cuki Cofresco SpA) for $3 \mathrm{~min}$, and the emitted scent was trapped for $2 \mathrm{~min}$ in a thermal desorption (TD) tube using a vacuum pump (G12/01 EB, Rietschle Thomas) with a flow rate of $200 \mathrm{~mL} / \mathrm{min}$. TD tubes were filled with $1.5 \mathrm{mg}$ of Carbotrap B and $1.5 \mathrm{mg}$ of Tenax-TA (both Supelco). To discriminate the scent of inflorescences from that of leaves, VOCs from 10 leaves were collected in situ from different individuals (one sample per taxon). Samples from empty oven bags were collected to identify ambient contaminants $(N=3)$. TD tubes were kept inside glass vials and stored at $-20^{\circ} \mathrm{C}$ until their chemical analysis.

\section{Chemical Analysis}

VOCs trapped in the TD tubes were analyzed using an automatic thermal desorption system (TD-20, Shimadzu, Kyoto, Japan) coupled with a Shimadzu GC/MS QP2010 Ultra equipped with a ZB-5 fused silica column (5\% phenyl polysiloxane; $60 \mathrm{~m}$, inner diameter $0.25 \mathrm{~mm}$, film thickness $0.25 \mu \mathrm{m}$, Phenomenex). The samples were run with a split ratio of $1: 1$ and a constant helium carrier gas flow of $1.5 \mathrm{~mL} / \mathrm{min}$. The oven temperature of the gas chromatograph $(\mathrm{GC})$ started at $40^{\circ} \mathrm{C}$, then increased by $6^{\circ} \mathrm{C} / \mathrm{min}$ to $250^{\circ} \mathrm{C}$ and held for $1 \mathrm{~min}$. The interface to the mass spectrometer (MS) worked at $250^{\circ} \mathrm{C}$. Mass spectra were taken at $70 \mathrm{eV}$ (electron ionization) from 30 to $350 \mathrm{~m} / \mathrm{z}$ (mass-to-charge ratio). GC/MS data were processed using the GCMSolution package, version 2.72 (Shimadzu). Identification of compounds was carried out using the NIST 11, Wiley 9, FFNSC 2, and Adams (2007) databases and the database available in MassFinder 3. To determine the absolute amount of scent emitted from an inflorescence, known amounts of terpenoids, aliphatics, and aromatics were injected into the GC/MS system; mean peak areas of these compounds were used to determine the total amount of scent (see Dötterl et al. 2005).

\section{Statistical Analysis}

To test for differences in the total emission rates of scent released by inflorescences of the parents and their hybrid, we used nonparametric Kruskal-Wallis (K-W) ANOVA (Statistica, ver. 7.1). The Tukey-Kramer test for nonparametric data was used as post hoc test, again using Statistica.

To compare the scent profiles of inflorescences and leaves among the taxa, we used qualitative (presence/absence of compounds) approaches using Primer, version 6.1.6 (Clarke and Gorley 2015). To determine pairwise qualitative similarities 
among samples, we calculated Sørensen's index. Based on the obtained similarity matrix, we performed analyses of similarities $(10,000$ permutations) to test for differences in the inflorescence scents between the parental taxa and the hybrid. Qualitative differences among inflorescence and leaf scent samples were represented with nonmetric multidimensional scaling, using Sørensen's index. Bray-Curtis similarities, based on the squareroot-transformed (to prevent the main components from having too strong of an influence on the outcome) percentage amount of compounds, were calculated to determine semiquantitative similarities among the samples. The obtained similarity matrix was analyzed, again in Primer, using canonical analysis of principal coordinates (CAP; Anderson et al. 2008), which provides a constrained ordination (discriminant analysis) to maximize the differences in scent among the taxa. To determine scent components that contributed most to the ordination pattern, vectors were overlaid to visualize relationships between the scent variables and the three taxa (Anderson et al. 2008). To test whether there were significant differences among the taxa in multivariate CAP space, a permutation test (10,000 permutations) was performed (Anderson et al. 2008).

\section{Results}

\section{Total Emission Rates of Scent}

Inflorescences of the parental taxa and their hybrid emitted different rates of scents $\left(\mathrm{K}-\mathrm{W}: \chi^{2}=13.00, \mathrm{df}=2, P=0.001\right.$; fig. 1). Post hoc analyses revealed that Calendula maritima $\left(\right.$ mean $=16.1 \pm 5.2 \mu \mathrm{g} \mathrm{h}^{-1}$ inflorescence ${ }^{-1}$ ) emitted a higher amount of scent than C. fulgida $\left(3.4 \pm 0.5 \mu \mathrm{g} \mathrm{h}^{-1}\right.$ inflorescence $^{-1}$ ), whereas the amount of scent released from the hybrid $\left(7.8 \pm 1.7 \mu \mathrm{g} \mathrm{h}^{-1}\right.$ inflorescence $\left.{ }^{-1}\right)$ was intermediate and not different from any parental taxa (fig. 1). From the leaves, we

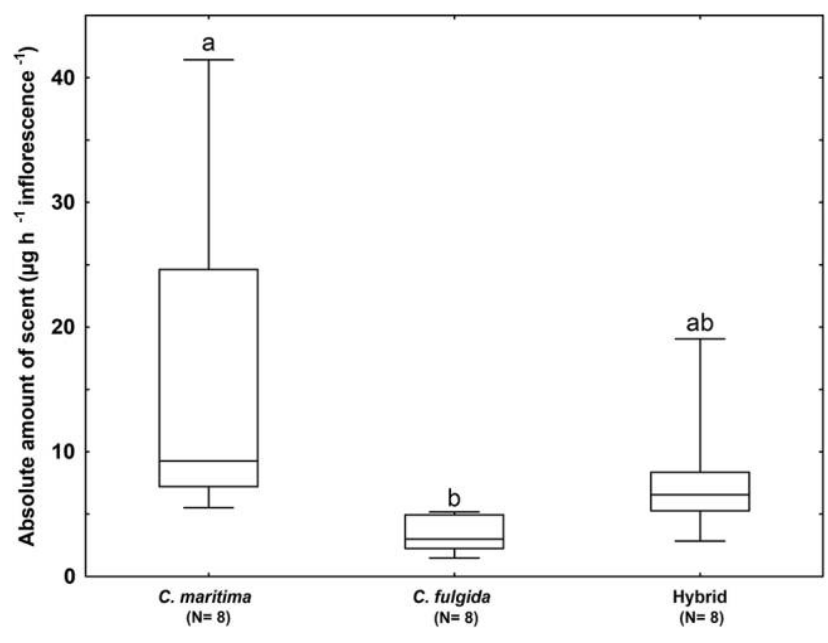

Fig. 1 Total emission rates of scent emitted from inflorescences of the two parental taxa (Calendula maritima and Calendula fulgida) and their hybrid. Groups with the same letter are not significantly different (Kruskal-Wallis ANOVA, Tukey-Kramer for nonparametric data). trapped between $1.3 \mu \mathrm{g} \mathrm{h}^{-1}$ leaf $^{-1}$ (hybrid) and $4.3 \mu \mathrm{g} \mathrm{h}^{-1}$ leaf $^{-1}$ (C. maritima).

\section{Qualitative Scent Profiles}

Overall, 32 compounds from three different chemical classes were found in the inflorescence scent samples: monoterpenes (16), sesquiterpenes (13), and aliphatic compounds (3; table 1). The sampled inflorescences of C. maritima emitted 29 VOCs, those of C. fulgida emitted 16 VOCs, and those of their hybrid emitted 19 VOCSs (table 1). Among these, 13 compounds (11 monoterpenes, one aliphatic compound, and one sesquiterpene) were shared by the three taxa. Calendula maritima inflorescences emitted 13 compounds, mainly sesquiterpenes, which were not found in the inflorescences of $C$. fulgida nor of the hybrid. The inflorescences of the hybrid had three compounds ( $\beta$-myrcene, $\alpha$-terpinene, and $\beta$-caryophyllene) in common with C. maritima that were absent in C. fulgida and three compounds in common with C. fulgida ((E)-2-hexenol, (Z)-3-hexenyl acetate, and $\alpha$ terpinolene) that were absent in C. maritima. Calendula maritima and C. fulgida did not have compounds in common that were absent in the hybrid. Overall, the hybrid released a spectrum of compounds more similar to that of C. fulgida than to that of C. maritima, whereas there were overall strong differences in qualitative scent properties among taxa $\left(R_{2,21}=0.99 ; P<0.001\right.$; fig. $2 A$ ), with every taxa differing from the others as indicated by post hoc analyses $(R \geq 0.95 ; P<0.01)$.

All compounds found in the inflorescence scents of the three taxa were also detected in the vegetative samples with one exception, i.e., $(Z)$ - $\beta$-ocimene, which was found only in inflorescence scent samples of C. maritima and did not occur in any vegetative sample. Generally, vegetative scent samples contained almost the same compounds as corresponding inflorescence scent samples in the three taxa (table 1), as also indicated by our ordination approach, in which vegetative samples were close to the inflorescence samples of respective taxa (fig. 2A).

\section{Semiquantitative Scent Profiles}

Similar to the spectrum of compounds, the hybrid also released a relative scent profile more similar to C. fulgida than to C. maritima, as indicated by a CAP analysis (fig. $2 B$ ), whereas there also were overall strong differences in the relative amount of scent among taxa (CAP, trace test $=1.39 ; P<0.001)$. Indeed, with the exception of one sample (original, C. fulgida, classified as hybrid), all samples were correctly classified (misclassification error $=4.17 \%$ ), showing that the three taxa also have distinct semiquantitative scent profiles. The four compounds $\beta$-bourbonene, $\alpha$-copaene, sativene, and $\alpha$-terpineol, which all were exclusive for C. maritima, were most responsible for ordination of samples along the axes. Nevertheless, the scent of all three taxa was dominated by the same three monoterpenes, i.e., sabinene, $\beta$-pinene, and limonene, which together constituted $62 \%$ (C. maritima), $71 \%$ (hybrid), and $76 \%$ (C. fulgida) of the total scent (table 1). Generally, the scent of all three taxa was strongly dominated by monoterpenes, with other substance classes contributing only relatively small relative amounts to the total scent (table 1). Monoterpenes, and, additionally, in C. maritima, aliphatic compounds, also dominated the three vegetative scent 
Table 1

Relative Amounts (\%) of Inflorescence and Leaf Scent Detected in Calendula maritima, Calendula fulgida, and Their Hybrid

\begin{tabular}{|c|c|c|c|c|c|c|}
\hline \multirow[b]{2}{*}{ Compounds, Kovats indexes } & \multicolumn{2}{|l|}{ C. maritima } & \multicolumn{2}{|l|}{ C. fulgida } & \multicolumn{2}{|l|}{ Hybrid } \\
\hline & $\begin{array}{c}\text { Inflorescences } \\
(N=8 ; \text { mean } \pm \mathrm{SE})\end{array}$ & $\begin{array}{l}\text { Leaves } \\
(N=1)\end{array}$ & $\begin{array}{c}\text { Inflorescences } \\
(N=8 ; \text { mean } \pm \mathrm{SE})\end{array}$ & $\begin{array}{l}\text { Leaves } \\
(N=1)\end{array}$ & $\begin{array}{c}\text { Inflorescences } \\
(N=8 ; \text { mean } \pm \mathrm{SE})\end{array}$ & $\begin{array}{c}\text { Leaves } \\
(N=1)\end{array}$ \\
\hline \multicolumn{7}{|l|}{ Aliphatic alcohols: } \\
\hline (Z)-3-hexenol, ${ }^{+} 855$ & $.2 \pm \operatorname{tr}$ & $\operatorname{tr}$ & $.9 \pm .3$ & 11.9 & $.3 \pm .1$ & 2.1 \\
\hline (E)-2-hexenol, ${ }^{+} 865$ & & & $.7 \pm .2$ & 2.1 & $.3 \pm .1$ & .9 \\
\hline 1-hexanol, ${ }^{+} 867$ & & & & .8 & & .2 \\
\hline \multicolumn{7}{|l|}{ Aliphatic esters: } \\
\hline (Z)-3-hexenyl acetate, ${ }^{+} 1005$ & & & $1.6 \pm .2$ & 17.8 & $.6 \pm .2$ & 3.0 \\
\hline \multicolumn{7}{|l|}{ Monoterpene alcohols: } \\
\hline Linalool, ${ }^{+} 1103$ & $.2 \pm .1$ & .1 & $.3 \pm .1$ & .2 & $.2 \pm \operatorname{tr}$ & .4 \\
\hline$\alpha$-terpineol, ${ }^{+} 1201$ & $1.2 \pm .2$ & .3 & & & & \\
\hline \multicolumn{7}{|l|}{ Monoterpene hydrocarbons: } \\
\hline$\alpha$-thujene, 933 & $3.0 \pm .7$ & 7.4 & $2.7 \pm .8$ & 1.5 & $2.8 \pm .3$ & 1.7 \\
\hline$\alpha$-Pinene, ${ }^{+} 941$ & $3.2 \pm .5$ & 5.7 & $8.0 \pm 2.4$ & 9.0 & $7.0 \pm .5$ & 1.7 \\
\hline Camphene, ${ }^{+} 958$ & & .2 & & & & \\
\hline Sabinene, ${ }^{+} 981$ & $34.3 \pm 2.7$ & 15.6 & $42.7 \pm 2.2$ & 5.2 & $35.7 \pm 1.6$ & 13.7 \\
\hline$\beta$-pinene, ${ }^{+} 986$ & $10.6 \pm 1.3$ & 7.5 & $14.2 \pm 1.5$ & 2.3 & $17.1 \pm .8$ & 2.5 \\
\hline$\beta$-myrcene, ${ }^{+} 993$ & $8.3 \pm .8$ & 7.4 & & 5.0 & $7.4 \pm 1.6$ & 6.2 \\
\hline$\alpha$-phellandrene, ${ }^{+} 1012$ & $1.3 \pm .4$ & 5.5 & $2.4 \pm .4$ & 4.7 & $1.9 \pm .2$ & 3.5 \\
\hline$\delta$-3-carene, 1018 & $.2 \pm \operatorname{tr}$ & 1.2 & $.1 \pm \operatorname{tr}$ & .3 & $.2 \pm \operatorname{tr}$ & .5 \\
\hline$\alpha$-terpinene, ${ }^{+} 1025$ & $1.9 \pm .5$ & 4.9 & & & $1.7 \pm .3$ & .4 \\
\hline p-cymene, ${ }^{+} 1033$ & $3.0 \pm .5$ & 5.6 & $3.1 \pm .5$ & 2.6 & $2.4 \pm .3$ & 4.0 \\
\hline Limonene, $^{+} 1037$ & $17.2 \pm 1.6$ & 13.4 & $19.2 \pm 1.8$ & 34.4 & $18.4 \pm 2.1$ & 53.6 \\
\hline$\beta$-phellandrene, ${ }^{+} 1038$ & $.6 \pm .1$ & .4 & $.6 \pm .1$ & .1 & $.5 \pm .1$ & 2.6 \\
\hline$(Z)-\beta$-ocimene, ${ }^{+} 1040$ & $.8 \pm .2$ & & & & & \\
\hline$(E)$ - $\beta$-ocimene, ${ }^{+} 1052$ & & .6 & & .2 & & \\
\hline$\gamma$-terpinene, ${ }^{+} 1066$ & $2.8 \pm .5$ & 5.0 & $2.4 \pm .6$ & .3 & $2.6 \pm .4$ & .8 \\
\hline$\alpha$-terpinolene, ${ }^{+} 1097$ & & 3.6 & $.9 \pm .2$ & .7 & $.9 \pm .1$ & .8 \\
\hline \multicolumn{7}{|l|}{ Sesquiterpene hydrocarbons: } \\
\hline$\alpha$-cubebene, 1366 & $1.2 \pm .2$ & .7 & & & & \\
\hline Unknown m/z: 105, 161, 93, 94, 106, 119 & $.4 \pm .1$ & .6 & & & & \\
\hline Unknown m/z: $105,94,161,119,204,120$ & $.6 \pm .1$ & .8 & & & & \\
\hline$\alpha$-copaene, 1396 & $2.3 \pm .5$ & 4.1 & & & & \\
\hline$\beta$-bourbonene, 1408 & $1.9 \pm .5$ & 2.5 & & & & \\
\hline Sativene, 1415 & $.5 \pm .1$ & .6 & & & & \\
\hline$\alpha$-gurjunene, 1434 & $.6 \pm .1$ & 1.2 & & & & \\
\hline$\beta$-caryophyllene, ${ }^{+} 1446$ & $.3 \pm .1$ & 1.1 & & .3 & $\operatorname{tr} \pm \operatorname{tr}$ & .6 \\
\hline Unknown m/z: $161,105,91,119,120,81$ & $.3 \pm .1$ & .5 & & $\operatorname{tr}$ & & \\
\hline Unknown m/z: 93, 107, 121, 105, 91, 79 & & .1 & & & & \\
\hline$\alpha$-caryophyllene, ${ }^{+} 1480$ & $.8 \pm .2$ & 1.6 & & & & \\
\hline$\beta$-selinene, 1514 & $1.1 \pm .3$ & 1.0 & $.2 \pm \operatorname{tr}$ & .7 & $.1 \pm \operatorname{tr}$ & .7 \\
\hline$\gamma$-cadinene, 1520 & $.5 \pm .2$ & .3 & & & & \\
\hline Unknown m/z: 81, 80, 93, 107, 147, 79 & & .1 & & .1 & & .2 \\
\hline$\delta$-cadinene, 1543 & $.7 \pm .2$ & .6 & & & & .1 \\
\hline Total no. compounds & 29 & 33 & 16 & 22 & 19 & 22 \\
\hline
\end{tabular}

Note. A plus sign indicates that authentic standards were available for identification; other compounds were identified based on published data for both mass spectrum and Kovats retention indexes (KI); compounds belonging to the same chemical class and functional group are arranged according to $\mathrm{KI}$; traces $(\mathrm{tr})=<0.05 \%$. 


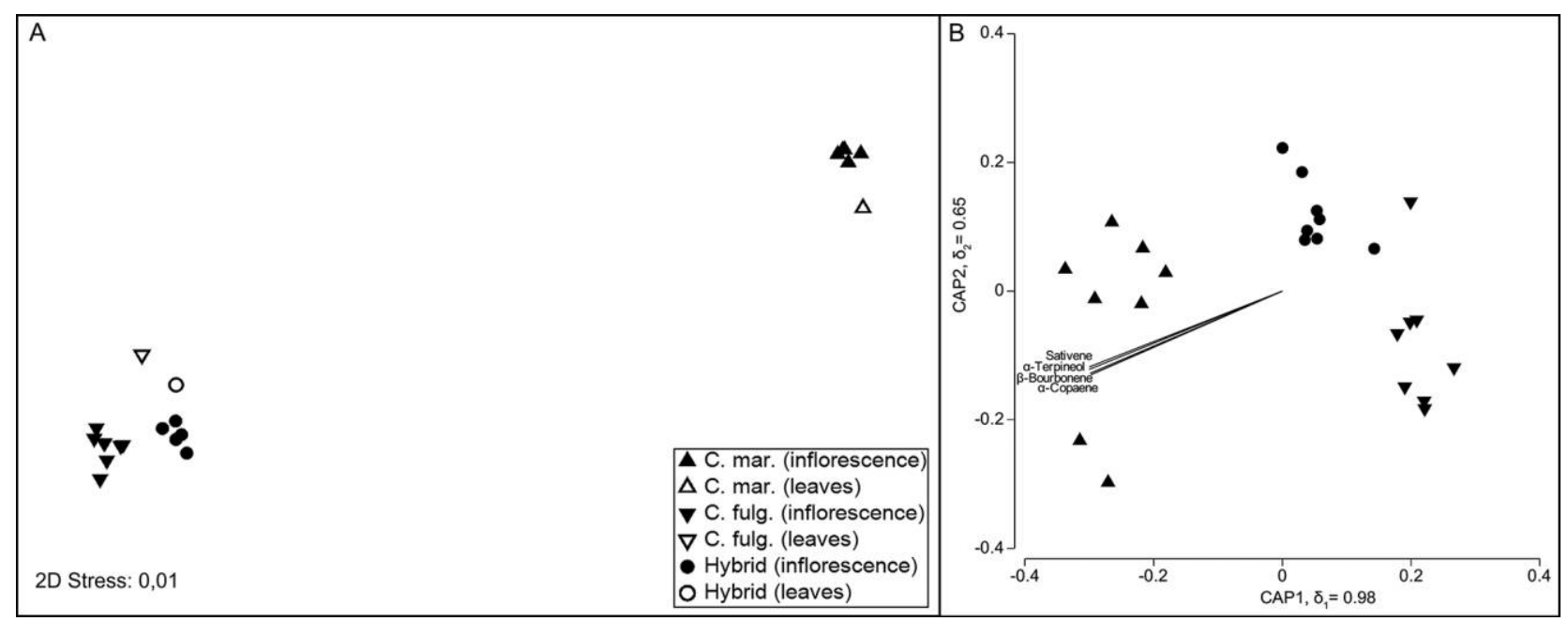

Fig. 2 Nonmetric multidimensional scaling, based on a Sørensen similarity matrix $(A)$, and canonical analysis of principal coordinates plot $(B)$ to display qualitative $(A)$ and semiquantitative $(B)$ differences in inflorescence and vegetative (only in $A$ ) scent profiles of two parental taxa (C. mar. = Calendula maritima, C. fulg. $=$ Calendula fulgida $)$ and their hybrid. The stress value of 0.01 and the large canonical correlations $\left(\delta_{1}=0.98, \delta_{2}=0.65\right)$ indicate that the ordinations are good representations of the observed similarities among the scent samples and that the multivariate data cloud and hypothesis of group differences are strongly associated, respectively. The four compounds correlating best with the coordinates are also given.

samples (table 1), with some differences in the relative amount of single compounds when compared to the inflorescence scent samples.

\section{Discussion}

The scent analyses showed that the inflorescence scents differ between parental Calendula fulgida and C. maritima and also between the parents and their hybrid. The differences in scent properties among the taxa are both qualitative and semiquantitative (fig. $2 A, 2 B$ ), with the hybrid being more similar to $C$. fulgida. Parental species also release different total amounts of scents, whereas the hybrid releases intermediate amounts not different from the two parental taxa (fig. 1).

Insects are believed to be involved in crossings as well as backcrossings of Calendula hybrids and their parents (Plume et al. 2013); however, to date, there is a big gap in the knowledge of inflorescence visitors of these taxa. During our scent collections, we performed preliminary flower visitor observations and recorded 12 bees of a single morphotype visiting the inflorescences of the three taxa-five on C. maritima (see also fig. 3), three on C. fulgida, and four on their hybrid. Four thereof were captured (one or two individuals per taxon), and all were identified as Panurgus siculus Mor. (Andrenidae; one female, three males). Bees of this genus are specialized on Asteraceae species for pollen collection (Shebl et al. 2015), and $P$. calcaratus was described to visit inflorescences of Calendula (P. calcaratus on C. maroccana and C. officinalis; Münze et al. 2006). Despite the need for further data, we speculate that the attraction of at least $P$. siculus to the three studied taxa may be linked with the yellow to orange inflorescence colors (visual cues) as well as to VOCs (olfactory cues), many of which are common to all taxa. The most abundant VOCs emitted from the three studied taxa (in both the number of compounds and the relative amount of the scent) were terpenoids, which are common among plants pollinated by bees (Schiestl 2010; Filella et al. 2013). More specifically, some of the compounds identified in the present study (e.g., linalool and $(E)-\beta$-ocimene) are even biologically active in andrenid bees, such as Andrena (Jürgens et al. 2008; Dötterl and Vereecken 2010), suggesting that they might be involved in host-plant location of $P$. siculus. Interestingly, terpenoids were identified as putative sex pheromones in Panurgus (Ayasse et al. 2001), suggesting that these bees have an attraction bias for such compounds.

Generally, in pollination biology, volatiles emitted specifically by flowers are considered to be important pollinator attractants (Raguso 2008). However, vegetative volatiles may also contribute to plant-pollinator interactions or even take over the signaling function from the flowers (Dufaÿ et al. 2003). In the present study, we found that in all three taxa, the single leaf scent samples contained all compounds present in the inflorescence scent samples (except $(Z)$ - $\beta$-ocimene, which was found only in C. maritima inflorescences), with overall a very similar qualitative and semiquantitative composition (table 1; fig. $2 \mathrm{~A}$, $2 B)$. The lack of inflorescence-specific VOCs suggests that both inflorescence and vegetative parts of Calendula may contribute to long-distance attraction of pollinators, which then might locate the inflorescence itself mainly by visual inflorescence cues and less by possibly minute differences in scent between vegetative and inflorescence parts. However, behavioral experiments are needed to determine the cues used by $P$. siculus for finding flowering Calendula plants and the cues they use to discriminate between flowering and nonflowering plants. Also, it should be verified whether the vegetative scents of these three taxa differ be- 

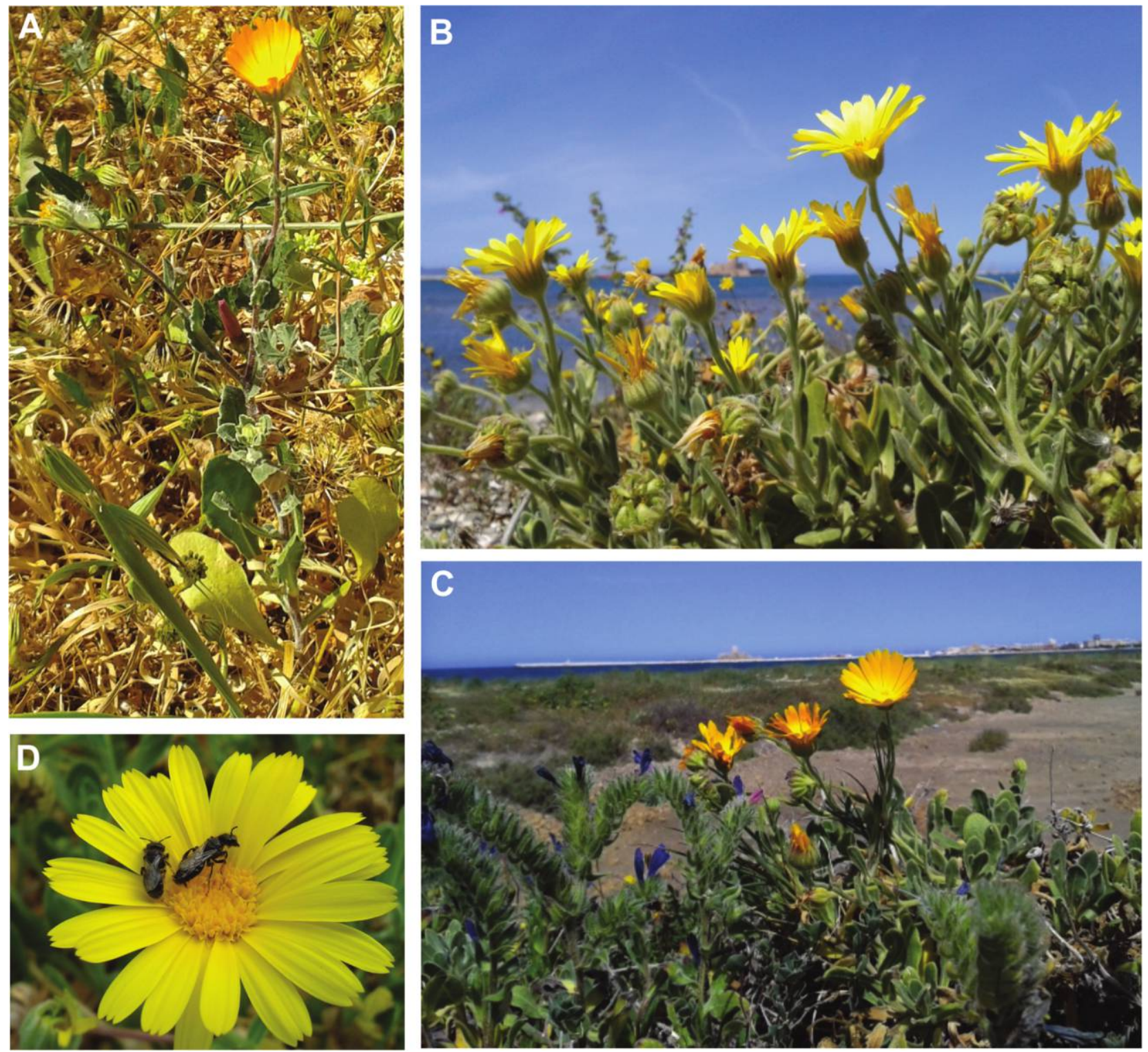

Fig. 3 Plants and inflorescence visitors. A, Calendula fulgida with orange flowers and not succulent leaf with irregularly dentate margin. $B$, Calendula maritima with yellow flowers and succulent leaf with entire margin. C, Hybrid between the two species (C. maritima $\times$ C. fulgida) with orange flowers and succulent leaf with entire margin. D, Panurgus siculus bees visiting an inflorescence of C. maritima.

tween flowering and nonflowering periods and whether they are mainly released during flowering as shown by Dufaÿ et al. (2003) for a palm.

Although Plume et al. (2013) highlighted that the hybrids between the two parental Calendula have a low fitness in natural habitat, it increases in human-disturbed areas such as coastal areas (F. Carimi, personal observation). In such habitats, the studied hybrid may outcompete C. maritima. Thus, hybridization with C. fulgida may represent a major threat for the relict populations of C. maritima, and this study is the first step toward understanding the lack of reproductive isolation in some populations of Calendula. However, further studies are necessary to identify the specific floral traits involved in the attraction of $P$. siculus and to determine the importance of these bees and potentially further flower visitors in transferring pollen within and among the three taxa of Calendula.

The finding that the hybrid emits intermediate amounts of scent with respect to its parents and that it produces a composition more similar to that of one of its parents is in concordance with Chartier et al. (2016). These authors found that hybrids between Arum italicum and A. maculatum emit intermediate scent profiles but with a composition closer to that of A. italicum. Diaz and Kite (2006) also reported that hybrids between $A$. creticum and A. idaeum have an intermediate scent composition more similar to one of their parents (A. creticum). So far, however, little is known about the (ge- 
netic) factors responsible for the hybrid's intermediate phenotypic traits that are often more similar to one of its parents.

Further studies are required in Calendula to verify the phenotypes and associated genotypes and to better understand the exact origin of the natural hybrids as well as the phenotypic and ecological characterization of $F_{1}$ hybrids obtained from controlled manual crossings or by molecular analyses. Unfortunately, for the genus Calendula, there are still no reliable nuclear genetic markers available (codominant molecular markers such as simple sequence repeat markers), making it very difficult to determine whether the hybrids are $\mathrm{F}_{1}, \mathrm{~F}_{2}$, or backcrosses. Therefore, in the present study, it was not possible to discriminate between F1 hybrids and advanced hy- brid generations, making the inclusion of both in our analyses possible.

\section{Acknowledgments}

We thank Irmgard Schäffler for supporting the scent analyses and for constructive discussions and Fritz Gusenleitner and Martin Schwarz for making the reference collection of bees at the Biology Center of Linz available. We are also grateful to the Klorane Foundation (France) for the financial support during the achievement of the Project "Salvaguardia delle popolazioni di Calendula maritima Guss., specie minacciata della flora siciliana."

\section{Literature Cited}

Adams RP 2007 Identification of essential oil components by gas chromatography/mass spectrometry. 4th ed. Allured, Carol Stream, IL.

Anderson M, R Gorley, K Clarke 2008 Permanova+ for Primer: guide to software and statistical methods. Primer-E, Plymouth, UK.

Ayasse M, RJ Paxton, J Tengö 2001 Mating behavior and chemical communication in the order Hymenoptera. Annu Rev Entomol 46:31-78.

Bischoff M, A Jürgens, DR Campbell 2014 Floral scent in natural hybrids of Ipomopsis (Polemoniaceae) and their parental species. Ann Bot 113:533-544.

Chartier M, S Liagre, H Weiss-Schneeweiss, B Kolano, JM Bessière, J Schönenberger, M Gibernau 2016 Floral traits and pollination ecology of European Arum hybrids. Oecologia 180:439-451.

Clarke KR, RN Gorley 2015 Primer V7: user manual/tutorial. PrimerE Ltd., Plymouth, UK.

Cortis P, NJ Vereecken, FP Schiestl, MR Barone Lumaga, A Scrugli, S Cozzolino 2009 Pollinator convergence and the nature of species' boundaries in sympatric Sardinian Ophrys (Orchidaceae). Ann Bot 104:497-506.

De Montmollin B, W Strahm 2005 The top 50 Mediterranean island plants: wild plants at the brink of extinction, and what is needed to save them. International Union for Conservation of Nature Species Survival Commission Mediterranean Islands Plant Specialist Group, Cambridge.

Diaz A, GC Kite 2006 Why be a rewarding trap? the evolution of floral rewards in Arum (Araceae), a genus characterized by saprophilous pollination systems. Biol 」 Linn Soc 88:257-268.

Dötterl S, NJ Vereecken 2010 The chemical ecology and evolution of bee-flower interactions: a review and perspectives. Can I Zool 88:668-697.

Dötterl S, LW Wolfe, A Jürgens 2005 Qualitative and quantitative analyses of flower scent in Silene latifolia. Phytochemistry 66:203-213.

Dufaÿ M, M Hossaert-McKey, MC Anstett 2003 When leaves act like flowers: how dwarf palms attract their pollinators. Ecol Lett 6:28-34.

Fennane M, MI Tattou 1998 Catalogue des plantes vasculaires rares, menacées ou endemiques du Maroc. Bocconea 8:5-243. (In French.)

Filella I, C Primante, J Llusià, AMM Gonzàlez, R Seco, G FarréArmengol, A Rodrigo, J Bosch, J Penuelas 2013 Floral advertisement scent in a changing plant-pollinators market. Sci Rep UK 3: 3434.

Hegarty MJ, SJ Hiscock 2005 Hybrid speciation in plants: new insights from molecular studies. New Phytol 165:411-423.
Jürgens A, S Dötterl, S Liede-Schumann, U Meve 2008 Chemical diversity of floral volatiles in Asclepiadoideae-Asclepiadeae (Apocynaceae). Biochem Syst Ecol 36:842-852.

Kölreuter JG 1761 Vorlaufige Nachrichten von einigen das Geschlecht der Pflanzen betreffenden Versuchen und Beobachtungen. Gleditschischen Handlung, Leipzig. (In German.)

Lanza D 1919 Monografia del genere Calendula L. Scuola Tip. "Boccone del Povero," Palermo (Estratto dagli Atti della Reale Accademia di Scienze, Lettere e Belle Arti di Palermo. Vol XI). (In Italian.)

Münze R, D Langner, M Nuß 2006 Die Bienenfauna des Botanischen Gartens Dresden (Hymenoptera: Apidae). Sachsische Entomol Z 1:45-69. (In German.)

Plume O, FM Raimondo, A Troìa 2013 Hybridization and competition between the endangered sea marigold (Calendula maritima, Asteraceae) and a more common congener. Plant Biosvst 149:6877.

Raguso RA 2008 Wake up and smell the roses: the ecology and evolution of floral scent. Annu Rev Ecol Evol Syst 39:549-569.

Rizzotto M 2003 Numeri cromosomici per la flora italiana: 14421445. Inf Bot Ital 35:95-97. (In Italian.)

Salzmann CC, FP Schiestl 2007 Odour and colour polymorphism in the food-deceptive orchid Dactylorhiza romana. Plant Syst Evol 267:37-45.

Schatz B, A Geoffroy, B Dainat, JM Bessière, B Buatois, M HossaertMcKey, MA Selosse 2010 A case study of modified interactions with symbionts in a hybrid Mediterranean orchid. Am _ Bot 97: 1278-1288.

Schiestl FP 2010 The evolution of floral scent and insect chemical communication. Ecol Lett 13:643-656.

Shebl MA, S Patiny, D Michez 2015 Supplementary note on the solitary bee fauna from the Suez Canal region of Egypt (Hymenoptera: Apoidea). I Melittology 47:1-5.

Troìa A, S Pasta 2006 Calendula maritima. International Union for Conservation of Nature Red List of threatened species 2006. doi:e.T61618A12524417.

Vereecken NJ, S Cozzolino, FP Schiestl 2010 Hybrid floral scent novelty drives pollinator shift in sexually deceptive orchids. BMC Evol Biol 10:103.

Waser N 2001 Pollinator behavior and plant speciation: looking beyond the "ethological isolation" paradigm. Pages 318-335 in L Chittka, JD Thomson, eds. Cognitive ecology of pollination. Cambridge University Press, New York. 